\title{
Technique of Specific Conductivity Measurement of Anisotropic Semiconductor Plates and Films
}

\author{
V.V. Filippov ${ }^{a, b, *}$, S.V. MitsuK $^{a}$, S.E. Luzyanin ${ }^{a}$ And V.P. Tigrov ${ }^{a}$ \\ ${ }^{a}$ Lipetsk State Pedagogical University, Lenina Str. 42, Lipetsk 398020, Russian Federation \\ ${ }^{b}$ Lipetsk State Technical University, Moskovskaya Str. 30, Lipetsk 398600, Russian Federation
}

Received: 23.05.2018 \& Accepted: 17.07.2020

Doi: $10.12693 /$ APhysPolA.138.759

*e-mail: wwfilippow@mail.ru

\begin{abstract}
The paper presents a fast technique to identify the components of specific conductivity tensor of thin semiconductor planes. The proposed technique is based on a well-known method with a linear location of a four-probe tester. It is scientifically backed up by solving the relevant boundary problems of electrodynamics and characterized by the simplicity of measurements and calculations. Moreover, the suggested method does not require the use of complicated equipment and also the boundary conditions have been taken into account. Experimental testing has been carried out on monocrystals of diarsenide of cadmium and diarsenide of zinc. Practical recommendations are given on how to make the necessary measurements more precise.
\end{abstract}

topics: anisotropic semiconductor, electroconductivity tensor, potential distribution, diarsenide of cadmium, fourprobe method

\section{Introduction}

Researchers now have a thorough study of methods available to characterize semiconducting materials which do not show anisotropy of physical properties [1, 2]. At the same time, in modern electronics promising semiconducting compounds of the type $\mathrm{A}_{2} \mathrm{~B}_{5}$ and $\mathrm{A}_{2} \mathrm{~B}_{6}$ are more and more widely used [3-6].

In crystals, we observe anisotropy of electric, thermoelectric and galvanomagnetic properties due to the complexity of crystal lattice. In some cases, stimulated anisotropy of electric properties of atomic semiconductors under pressure or under the influence of external field can also be observed $[5,6]$. As a consequence, the development of anisotropic semiconductor characterization methods becomes ever more important.

Probe methods $[1,2]$ confirmed their efficiency in measuring specific electroconductivity. Difficulty of such measurements consists in the fact that a current spreading effect appears in these materials, depending on a correlation of the value of conductivity tensor. That is why the methods which are welldeveloped and sufficient for isotropic materials are not applicable to anisotropic ones.

Scientific literature describes methods intended for specific conductivity measurement like the Montgomery method [7]. Its essence lies in a numerical identification of electric potential distribution on the lateral lanes of the sample. The Montgomery method puts rigid requirements on the sample geometry and quality of the lateral planes. The method has proved its efficiency for rectangular samples and only in such a case it is possible to create high quality low-resistance contacts on the flat lateral planes, although it is not always possible to achieve it in practice. A certain problem arises when one needs to analyze conductivity but the lateral planes are not available or there are difficulties with an application contact place. In fact, there are some methods [8] which are further derived from the Montgomery method. Such methods increase the accuracy of a specific conductivity measurement, however they still set high requirements for the quality of surfaces of the lateral planes and necessitate the geometrical location of the contacts at the sample angles.

Quantitative methods identifying specific conductivity tensor of anisotropic samples were already described in scientific literature [9-11]. However, they do not allow to identify the required values with high accuracy and they are characterized by an error of 10-15\%. Such methods require lengthy resistance contacts, the creation of which often results in the destruction of a semiconductor crystal which renders it unfit for future work.

This paper suggests a technique to identify the components of specific conductivity tensor of anisotropic planes and films which is based on a method with a linear location of a four-probe tester. This technique is also assessed in terms of its application and measurement accuracy. 


\section{Theoretical background of the technique}

Let us identify the potential distribution with probe measurement when the current is permanent. In the fixed mode with absent sources and drain charge, the vector of current density $\boldsymbol{j}$, the intensity of field $\boldsymbol{E}$ and the potential $\varphi$ are related to each other in the following way [12]:

$$
\boldsymbol{j}=\hat{\sigma} \boldsymbol{E}, \quad \boldsymbol{E}=-\nabla \varphi, \quad \nabla \cdot \boldsymbol{j}=0,
$$

where $\hat{\sigma}$ is the symmetrical tensor of specific conductivity. For the sample oriented, in particular, in a way that the tensor has a diagonal shape

$$
\tilde{\sigma}=\left(\begin{array}{ccc}
\sigma_{x} & 0 & 0 \\
0 & \sigma_{y} & 0 \\
0 & 0 & \sigma_{z}
\end{array}\right),
$$

we can get the differential equation for the potential:

$$
\sigma_{x} \frac{\partial^{2} \varphi}{\partial x^{2}}+\sigma_{y} \frac{\partial^{2} \varphi}{\partial y^{2}}+\sigma_{z} \frac{\partial^{2} \varphi}{\partial z^{2}}=0
$$

One should take into account that the normal constituent of the current density on the sample surface is equal to zero everywhere except for the points under the current electrodes [12]. For the probes located according to Fig. 1, the following boundary conditions apply:

$$
\begin{aligned}
& \left.\frac{\partial \varphi}{\partial x}\right|_{x=0, a}=0,\left.\quad \frac{\partial \varphi}{\partial y}\right|_{y=0, b}=0,\left.\quad \frac{\partial \varphi}{\partial z}\right|_{z=0}=0, \\
& \left.\frac{\partial \phi}{\partial z}\right|_{z=d}=\frac{I_{14}}{\sigma_{z}} \delta\left(x-x_{1}\right) \delta\left(y-y_{1}\right) \\
& -\frac{I_{14}}{\sigma_{z}} \delta\left(x-x_{4}\right) \delta\left(y-y_{4}\right),
\end{aligned}
$$

where $\delta(x)$ is the Dirac delta-function. Its usage is justified for the current probes with small-size entry area. The coordinates of the current probes are $\left(x_{1}, y_{1}\right)$ and $\left(x_{4}, y_{4}\right)$.

The boundary problem of (3) and (4) is solved with the method of separation of variables. As a result, the final expression for potential $\varphi$ on the sample surface can be represented in the form of the double Fourier series

$$
\begin{aligned}
\varphi & =-\frac{4 I_{14}}{a b \sigma_{z}} \sum_{k, n=0}^{\infty} A_{n k} \frac{\operatorname{ch}\left(\eta_{k n}(d-z)\right)}{\eta_{k n} \operatorname{sh}\left(\eta_{k n} d\right)} \\
& \times \cos \left(\alpha_{k} x\right) \cos \left(\beta_{n} y\right)
\end{aligned}
$$

where

$$
\begin{aligned}
& A_{n k}=\Theta_{n} \Theta_{k} \cos \left(\alpha_{k} x_{1}\right) \cos \left(\beta_{n} y_{1}\right) \\
& -\Theta_{n} \Theta_{k} \cos \left(\alpha_{k} x_{4}\right) \cos \left(\beta_{n} y_{4}\right), \\
& \alpha_{k}=\frac{\pi k}{a}, \quad \beta_{n}=\frac{\pi n}{b}, \quad \eta_{k n}=\sqrt{\frac{\sigma_{x}}{\sigma_{z}} \alpha_{k}^{2}+\frac{\sigma_{y}}{\sigma_{z}} \beta_{n}^{2},} \\
& \Theta_{i\{i=k, n\}}= \begin{cases}1, & i \neq 0, \\
\frac{1}{2}, & i=0 .\end{cases}
\end{aligned}
$$

To identify the components of electroconductivity tensor $\sigma_{x}, \sigma_{y}$, it is necessary to carry out two

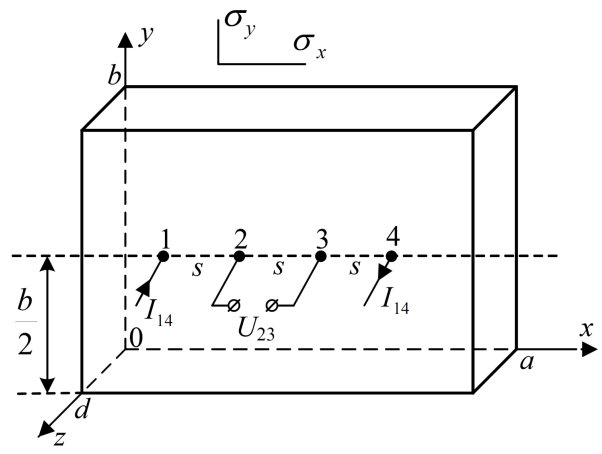

Fig. 1. The scheme of probe location on a rectangular sample.

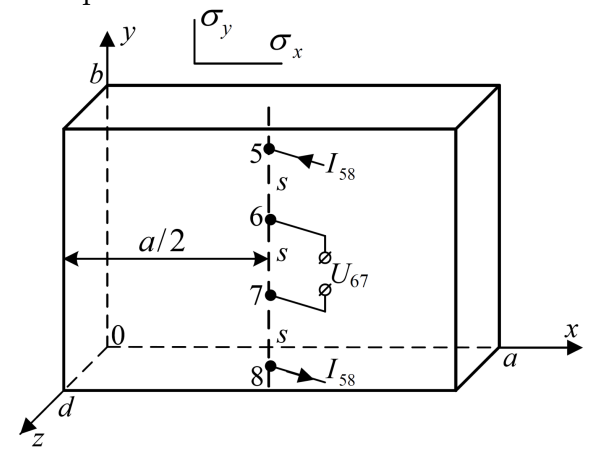

Fig. 2. The scheme of probe location on a rectangular sample.

independent measurements of current and voltage with different locations of probes. Firstly, we place a tester with a linear location of probes on the sample surface along the line which is parallel to axis $x$, then we measure current $I_{14}$ and potential difference $U_{23}$ (in Fig. 1: $x_{1}=(a-s) / 2, x_{4}=(a+3 s) / 2$, $\left.y_{1}=y_{4}=b / 2\right)$. The obtained potential distribution enables us to find the theoretical potential difference between probes 2 and 3 :

$$
\begin{aligned}
U_{23} & =\frac{I_{14} a}{\sigma_{x} b d} L_{1}, \\
L_{1} & =\frac{16 \sigma_{x}}{a^{2} \sigma_{z}} \sum_{\substack{k=1,3,5, \ldots, n=0,2,4, \ldots}} \Theta_{n} \frac{\operatorname{cth}\left(\eta_{k n} d\right)}{\eta_{k n}} \\
\times & \sin \left(\frac{1}{2} \alpha_{k} s\right) \sin \left(\frac{3}{2} \alpha_{k} s\right) .
\end{aligned}
$$

With the ratio value $d / s<0.7$, the sample can be considered to be thin with the calculation error of less than $2 \%[1,2]$. In this case, we can do summation over " $k$ " [13]. As a result, in the approximation of the thin sample we get the expression for multiplier $L_{1}$, included into (8), namely:

$$
\begin{aligned}
& L_{1}=\frac{s}{a}+\sum_{n=2,4,6 \ldots} \frac{2}{\gamma \beta_{n} a \operatorname{sh}\left(\gamma \beta_{n} a\right)} \\
& \quad \times\left[\operatorname{ch}\left(\gamma \beta_{n}(a-s)\right)-\operatorname{ch}\left(\gamma \beta_{n} s\right)\right. \\
& \left.\quad+\operatorname{ch}\left(2 \gamma \beta_{n} s\right)-\operatorname{ch}\left(\gamma \beta_{n}(a-2 s)\right)\right] .
\end{aligned}
$$

Here, $\gamma=\sqrt{\sigma_{y} / \sigma_{x}}$ reads as the parameter of electroconductivity anisotropy. 
For the second measurement, we place a probe on the same plane of the sample along the line parallel to axis $y$ (in Fig. 2: $x_{5}=x_{8}=a / 2, y_{5}=(b+3 s) / 2$, $\left.y_{8}=(b-3 s) / 2\right)$ and measure current $I_{58}$ and distribution potential $U_{67}$. Analogically, we get the expression for the correlation of current $I_{58}$ and voltage $U_{67}$ :

$$
U_{67}=\frac{I_{58}}{\sigma_{y}} \frac{b}{a d} L_{2},
$$

where $L_{2}$ is the geometrical factor corresponding particularly to the considered case of the probe location. In the case of thin samples we get:

$$
\begin{gathered}
L_{2}=\frac{s}{b}+\sum_{k=2,4,6 \ldots} \frac{2}{\alpha_{k} b / \gamma \operatorname{sh}\left(\alpha_{k} b / \gamma\right)} \\
\times\left[\operatorname{ch}\left(\alpha_{k}(b-s) / \gamma\right)-\operatorname{ch}\left(\alpha_{k} s / \gamma\right)\right. \\
\left.+\operatorname{ch}\left(2 \alpha_{k} s / \gamma\right)-\operatorname{ch}\left(\alpha_{k}(b-2 s) / \gamma\right)\right]
\end{gathered}
$$

Using the measurement data, we identify the value of the dimensionless parameter $Q$ :

$$
Q=\frac{R_{1}}{R_{2}}=\frac{U_{23} / I_{14}}{U_{67} / I_{58}},
$$

which we need to calculate the value $\gamma$, necessary for getting the value of the components of specific electroconductivity tensor.

According to (8) and (11), the "theoretical" relation $Q(\gamma)$ can be expressed in the following way:

$$
Q(\gamma)=\left(\frac{\gamma a}{b}\right)^{2} \frac{L_{1}}{L_{2}}
$$

\section{Technique of measurement procedure}

The suggested technique aimed at the measurement of components of special electroconductivity tensor of a crystal or film can be summarized as follows:

1. With a certain value of current $I_{14}$ measure voltage $U_{23}$, then using the same linear probe with current value $I_{58}$ measure voltage $U_{67}$ (see Fig. 1 and Fig. 2).

2. Calculate from the experiment the value of parameter $Q=R_{1} / R_{2}$ according to (13).

3. Using the relation plot $Q(\gamma)$ identify the value of parameter $\gamma$ by putting in correspondence the "theoretical" and experimental values of parameter $Q$ (obtained with (14) and (13)). For some values of relations $a / s$, $b / s$, we showed $Q$ related to anisotropy parameter $\gamma$ in Fig. 3 .

4. Identify the value of the geometrical factors $L_{1}$ and $L_{2}$ using (10) and (12).

5 . Calculate the value of a component of electroconductivity tensor $\sigma_{x}$ and $\sigma_{y}$ using:

$$
\sigma_{x}=\frac{I_{14}}{U_{23}} \frac{a}{d b} L_{1}, \quad \sigma_{y}=\frac{I_{58}}{U_{67}} \frac{b}{d a} L_{2} .
$$



Fig. 3. The diagram showing the relation of parameter $Q$ value to electroconductivity anisotropy coefficient $\gamma$ with $a=b$ and various values of $s$.

For practical considerations, it is worth to identify the value of geometrical factors $L_{1}$ and $L_{2}$ for the infinite sample $(a / s \rightarrow \infty, b / s \rightarrow \infty)$. As a result of calculations of the relevant limits, we conclude that for an indefinite plane the expressions (15) can be represented in the following way:

$$
\sigma_{x}=\frac{0.2206}{\gamma d t} \frac{I_{14}}{U_{23}}, \quad \sigma_{y}=\frac{0.2206 \gamma}{d} \frac{I_{58}}{U_{67}} .
$$

For isotropic samples $\left(\sigma_{x}=\sigma_{y}=\sigma, \gamma=1\right)$ we get a well-known formula used to calculate specific resistance when the measurements are done with the help of a linear four-probe tester $[1,2]$ :

$$
\rho=\frac{1}{\sigma}=4.532 \frac{U_{23}}{I_{14}} d
$$

\section{Experimental validation and conclusions}

The suggested technique aimed at identifying the components of specific electroconductivity tensor was experimentally tested on anisotropic $\mathrm{CdAs}_{2}$ and $\mathrm{ZnAs}_{2}$ [14, 15] plates. We used clamping wolframium probes as current and measurement contacts.

Constant electric current passed through the samples under study. The current came from stabilized power supply B5-44, a potential difference between measuring probes was measured by voltameter V2-34, the positions of measuring and current probes were monitored and controlled with the MBS-10 microscope. The average values of electroconductivity tensor are given in Tables I and II. The control values of these components measured by a standard two-probe method [1, 9] are also included for comparison. For the practical measurement of conductivity control values we used tinker contacts, the ohmic properties of which to diarsenide of cadmium and diarsenide of zinc were proved experimentally $[3,4]$.

One of restrictions that limit the use of the suggested measurement method is the size of the sample: the transverse size of semiconductors under study must be bigger than the length of a free path 
Results of experimental measurements.

TABLE I

\begin{tabular}{c|c|c|c|c|c|c|c|c}
\hline \hline \multirow{2}{*}{ Sample } & $a[\mathrm{~mm}]$ & $b[\mathrm{~mm}]$ & $d[\mathrm{~mm}]$ & $s[\mathrm{~mm}]$ & \multicolumn{2}{|c|}{$\sigma_{x}\left[(\Omega \mathrm{m})^{-1}\right]$} & \multicolumn{2}{|c}{$\sigma_{y}\left[(\Omega \mathrm{m})^{-1}\right]$} \\
\cline { 5 - 8 } & & & & $\begin{array}{c}\text { Proposed } \\
\text { method }\end{array}$ & $\begin{array}{c}\text { Two-probe } \\
\text { method }\end{array}$ & $\begin{array}{c}\text { Proposed } \\
\text { method }\end{array}$ & $\begin{array}{c}\text { Two-probe } \\
\text { method }\end{array}$ \\
\hline $\mathrm{CdAs}_{2}$ & 19.20 & 22.50 & 1.00 & 3.00 & 41.34 & 42.10 & 8.82 & 8.90 \\
$\mathrm{ZnAs}_{2}$ & 18.00 & 20.00 & 0.85 & 3.00 & 2009 & 2021 & 509 & 520
\end{tabular}

\section{TABLE II}

Comparison of experimental measurements and results obtained with other methods, i.e., (1) - Montgomery [7], (2) — modified Montgomery [8], (3) Schnabel [4], (4) — transverse [11].

\begin{tabular}{l|c|c|c|c|c|c|c|c}
\hline \hline \multirow{2}{*}{ Sample } & \multicolumn{4}{|c|}{$\sigma_{x}\left[(\Omega \mathrm{m})^{-1}\right]$} & \multicolumn{4}{|c}{$\sigma_{y}\left[(\Omega \mathrm{m})^{-1}\right]$} \\
\cline { 2 - 8 } & $(1)$ & $(2)$ & $(3)$ & $(4)$ & $(1)$ & $(2)$ & $(3)$ & $(4)$ \\
\hline $\mathrm{CdAs}_{2}$ & 38.25 & 39.72 & 37.33 & 44.14 & 8.05 & 8.14 & 8.03 & 9.32 \\
$\mathrm{ZnAs}_{2}$ & 1908 & 1926 & 1905 & 2181 & 442 & 464 & 443 & 561
\end{tabular}

of an electron. The second restriction is the presence of a flat upper plane, adjacent to the measurement contacts. Also, it is necessary for general resistance of the semiconductor $R$ (in the order of value) to meet the condition: $R_{\mathrm{A}} \ll R \ll R_{\mathrm{V}}$ (where $R_{A}$ - the resistance of amperemeter, $R_{V}$ — the resistance of voltmeter).

The advantage of the suggested method is that it is not necessary to make an ohmic connection to the sample - this procedure often creates certain difficulties leading to spoiling the crystal which becomes unsuitable for further use. The error of this technique is determined by the error of the wellknown four-probe method.

\section{References}

[1] D.K. Schroder, Semiconductor Material and Device Characterization, John Wiley \& Sons, Inc., 2006.

[2] W.R. Runyan, T.J Shaffner, Semiconductor Measurements and Instrumentation, McGraw Hill Professional, 1998.
[3] S.F. Marenkin, V.M. Truhan, Fosfidi, Arsenide Cinka $i$ Kadmiya (Phosphides, Arsenides of Zinc and Cadmium), Minsk 2010 (in Belarusian).

[4] P.A. Popov, E.A. Oleinik, V.M. Trukhan, A.D. Izotov, S.F. Marenkin, Inorg. Mater. 54, 237 (2018).

[5] I.G. Neizvestnyj, V.A. Gridchin, Russ. Microelectron. 38, 71 (2009).

[6] Shi-Quan Feng, Ling-Li Wang, Xiao-Xu Jiang, Hai-Nin Li, Xin-Lu Cheng, Lei Su, Chin. Phys. B 26, 046301 (2017).

[7] H.C. Montgomery, J. Appl. Phys. 42, 2971 (1971).

[8] C.A.M. dos Santos, A. de Campos, M.S. da Luz, B.D. White, J.J. Neumeier, B.S. de Lima, C.Y. Shigue, J. Appl. Phys. 110, 083703 (2011).

[9] P. Walmsley, I. R. Fisher, Rev. Sci. Instrum. 88, 043901 (2017).

[10] P. Schnabel, Z. Angew. Phys. 22, 136 (1967).

[11] G.A. Levin, J. Appl. Phys. 81, 714 (1997).

[12] L.D. Landau, E.M. Lifshitz, Electrodynamics of Continuous Media, Pergamon, Oxford 1984 .

[13] I.S. Gradshteyn, I.M. Ryzhik, Tables of Integrals, Series, and Products 6th ed., Academic Press, New York 2000.

[14] V.V. Filippov, S.V. Mitsuk, Chin. Phys. Lett. 34, 077201 (2017).

[15] V.V. Filippov, E.N. Bormontov, Semiconductors 47, 884 (2013). 